\title{
Improving Website Ranking through Search Engine Optimization
}

\author{
Ali H. Al-Badi ${ }^{1}$, Ali O. Al Majeeni ${ }^{2}$, Pam J. Mayhew ${ }^{2}$ and Abdullah S. Al-Rashdi ${ }^{3}$ \\ ${ }^{1}$ Information Systems Department, College of Commerce and Economics, \\ Sultan Qaboos University, Oman \\ ${ }^{2}$ School of Computing Sciences, University of East Anglia, Norwich, UK \\ ${ }^{3}$ Center for Information Systems, Sultan Qaboos University, Oman
}

\begin{abstract}
Internet is fast becoming critically important to commerce, industry and individuals. Perhaps the most important single component of this communication network is the Search Engine (SE). Successful Websites rank high in SEs hits, whereas unsuccessful Websites may not rank at all.

In an effort to increase the ranking of Sultan Qaboos University (SQU) amongst international universities, it was recommended to apply "Webometrics" measures. Hence, it was necessary to enhance the university's Website ranking. Therefore, the primary aim of this research is to explore the factors and techniques that contribute to a page ranking highly in a Search Engine Results Page (SERP). The research is divided into two parts. The first part is a descriptive/interpretive analysis aiming to provide an outline of the current and relevant literature with regard to Search Engine Optimization (SEO). It covers how SEs work, and explores the guidelines that are available for Web designers to optimize Web pages, thereby increasing their visibility and ranking. Furthermore, it provides a short history of the most important SEs, differentiates between them, and reviews the approaches they use to crawl, index, and display search results. It also provides an account of the different factors/methods impacting Website ranking. The second part documents an action research project undertaken to enhance the visibility and ranking of the SQU Website.
\end{abstract}

The main findings are that, for a site to rank well, it must use a combination of increasingly sophisticated techniques and tools, and not to rely on outdated methods such as Meta tags (especially the keywords). Fortunately, there are many online tools which can assist in tuning a site to work better, as well as other freely available good pieces of advice.

Keywords: Search Engine Optimization, Search Engine, crawl, index, user behavior, understanding target audience, Web usability, Website traffic, Web robots, Meta tags, Website Visitor Analysis, Search Engine Watch, Google AdWords

\section{Introduction and Background}

It has been said that "If Content is King, Quality is Queen" (Langlois, 2010), and this is particularly appropriate when applied to the Internet. The pair go hand in hand i.e. the Web content is worthless if it is not of a good quality. With seemingly endless content available online, the findability of appropriate information is critical. Search Engines (SEs) are the tools used to find this content, and they can be classified into three groups:

- General: e.g. Google, Yahoo, Bing (formerly Live Search, Windows Live Search, and MSN Search), and ask (formerly AskJeeves.com); 
- Vertical Search: specialist SEs that focus on specific resources reducing the number of irrelevant hits, e.g. Travelocity and Expedia;

- Location Based SEs: these use geographical information (such as zip codes or IP addresses) about the users to provide more relevant results.

Most of today's SEs search for Web pages utilizes a user supplied text sample (a keyword or phrase). The SE returns a list of "hits" or Web references that match the sample; these are displayed on the Search Engine Results Page (SERP), listed and sorted by relevance. Gathered information is not limited to the text, and can include images, PDF files and other types of content. SEs may rank hits differently; a hit ranked high by one could be ranked low by another. What is found and how it is ranked, is determined by the SE's algorithms, as well as the way keywords are presented to the SEs, and the way Web pages are designed to expose keywords to search processes.

So, how do SEs know what information is to be found on individual Web pages? They know by using software applications called "Web Robots"; these robots "crawl around" Internet Websites gathering data, which is then organized and indexed. Once this is done, the data can be searched quickly and easily. Since SEs do not all work in the same way, results may differ. Deitel provides an account of major SEs in his book (Deitel, 2008). Similarly, John Battelle's book "The Search: How Google and Its Rivals Rewrote the Rules of Business and Transformed Our Culture" (Battelle, 2006) provides an extensive history of SEs, and presents strong arguments for the importance of search in almost every aspect of our personal and business lives.

\section{Website Optimization and Marketing}

It is one of the primary goals of today's Web designers to maximize the position of their Web pages in SE rankings. If one wants a particular Website to be returned as a highly ranked hit, that site must be found by the Web robot, then fully descriptive information must be extracted and the site indexed. The process of designing and tuning a Website to maximize its findability and improve its ranking is known as "Search Engine Optimization" (SEO) (Fleischner, 2011; Kent, 2008; Interleado, 2009). There are two ways of employing SEO: firstly, white hat SEO, which refers to the legitimate methods supported by SEs; these methods do not attempt to deceive SEs, and they produce quality, long-term results. Techniques include: offering quality and relevant content, proper metadata and effective keywords, and having inbound links from other relevant high-quality pages. Secondly, black hat SEO, in which designers attempt to deceive SEs by manipulating their Web pages content to suggest that they are something other than what they actually are. Therefore, black hat SEO is not really optimization; it is more a form of SE deception (Wilding, 2009; Deitel, 2008).

The term SEO can also be applied to a new breed of companies, which seek to determine the most important factors to be used to make a Web page rank highly in SERPs (Evans, 2007). Table 1, below, shows some examples of this emerging industry.

Table 1: Search Engines Optimization Companies

\begin{tabular}{|c|l|l|}
\hline No & SEO company name & SEO company URL \\
\hline 1 & SEOMoz.org & http://www.seomoz.org/ \\
\hline 2 & HighRankings.com & http://www.highrankings.com/ \\
\hline 3 & SearchEngineWatch.com & http://searchenginewatch.com/ \\
\hline 4 & SearchEngineWorld.com & http://searchengineworld.com/ \\
\hline 5 & MarketLeap.com & http://marketleap.com/ \\
\hline 6 & Bruce Clay Inc & http://www.bruceclay.com/ \\
\hline 7 & SEO inc & http://www.seoinc.com/ \\
\hline
\end{tabular}


Despite the proliferation of SEO companies, and the individual efforts in this regard, the accumulated knowledge only represents partial information of SE's heuristics, based mainly on trial and error approach (Fortunato et al, 2006). Many previous studies (such as Ledford, 2009; Evans, 2007; Bifet et al., 2005; Moran and Hunt, 2006; Fortunato et al., 2006; Pringle et al., 1998; Khaki-Sedigh and Roudaki, 2003) have attempted to explore the factors that could potentially influence a SE's ranking of a Website. Obviously, SEs use a variety of mechanisms to rank Web pages (Sullivan, 2007; Evans, 2007). These factors have been classified into two main categories: QueryFactors, which are based on the content of a Web page, such as the existence and frequency of keywords in Title Tag, and Query-Independent Factors, which are based on information obtained from external Web pages that are linked to a Web page under consideration. However, all these factors are rather difficult to quantify as the SEs do not reveal which factors they use when determining a Website's ranking. Therefore, it is clear that to maximize one's Website ranking, it is not sufficient to rely upon any one mechanism or factor, and thus a number of measures must be adopted.

Another method whereby Website ranking may be improved is "Search Engines Marketing" (SEM). It is a method of promoting Websites to increase traffic and search results by raising the site's visibility on SEs result pages. SEM includes SEO, managing paid listings, developing online marketing strategies and submitting sites to directories (Deitel, 2008). Most SEs differentiate between paid and regular hits; for example, they may display paid results in a separate list, or with a different background highlight.

The following sections will provide insight into the factors, methods, and techniques used to enhance page ranking.

\section{Factors/Methods Impacting Website Ranking}

Perhaps at one time, when the Internet was young, getting one's site recognized and ranked highly was a simple matter. One method that was once thought to be effective was to use Meta tags. It was felt that SEs would give such tags emphasis in ranking sites, and this was a common misconception held by some, even today. Nowadays, things are much more complex. In this section we provide a review of Meta tags, detailing why they arose and how they fell from favor. This is followed by an overview of design factors that need to be considered when designing a site for high visibility and better ranking.

\section{Meta tags}

Meta tags are used to provide SEs with information that is not visible when the page is displayed in a browser (Sullivan, 2002); their role in improving Website visibility is often misunderstood. Meta tags go within the Web page's header. The header of any HTML file consists of the following components:

a. Title: this is usually shown in SE listings, in the title bar of the browser, and is used in bookmarks (favorites).

b. Description: SEs sometimes display this text in the SERP.

c. Meta Keywords: this tag is only supported by some major robots, and is used to provide supplementary information to Web robot directives.

d. Meta Robots: these are used to exclude some Web pages from being indexed. 


\section{Meta Tag History}

An Internet draft memo published in December 1995 (Sullivan, 2007) gives what is probably the earliest and most authoritative mention of Meta tags. Several major SE companies convened in May 1996 to discuss Meta tags (Sullivan, 2007), and that meeting gave birth to a common standard for Meta robots and the Meta description tags. As for the Meta keywords tag, no specification was agreed upon, but despite this, both Infoseek and Alta Vista offered support for the Meta keywords tag in 1996 (Sullivan, 2007). Inktome also provided support when it began operations later in 1996, and Lycos added support in 1997.

However, when new SEs such as Google and FAST emerged in 1998, they did not support the tag. This happened because SEs companies had learned that some webmasters would "stuff" the same word repeatedly into the Meta keywords tag so their sites would rank better. In 2002, due to this abuse, Alta Vista dropped its support for the tag; later, other major SEs also dropped their support. Now, SEs use cumulative factors to rank Web pages and the investigation of such factors is the subject of this research paper.

\section{Methods Used to Improve Website Visibility and Ranking}

Because of the importance of Website visibility, many different people and organizations have studied the factors/methods that influence a site's rank with major SEs (as outlined in section 1.1). The authors of this paper were surprised by the wealth of guidelines and tools that exist to help webmasters to create highly ranked Web pages. It was found that there are many factors influencing Website visibility including, but not limited to, Meta tags.

One of these methods is link building, which is a process of increasing SE ranking and traffic by generating inbound links to a particular Website. SE algorithms regard each link as a vote for the destination Website's content; therefore, sites with the greatest number of high quality inbound links appear the highest in SERPs. Deitel (2008) stated that there are three main methods of building links, which are: a)
Reciprocal linking, which is an exchange in which two related Websites (such as two universities) link to each other; b) Link baiting, which involves creating attentiongrabbing Web content for the purpose of increasing exposure through employing Social Media and Social Bookmarking Websites; and c) Natural linking, which is the process of building one-way inbound links by optimizing Website content and user experience without the explicit solicitation of a reciprocal link (Deitel, 2008).

SEOmoz is one of SEO companies specializing in the development of resources for maximizing SEO. In 2009, SEOmoz highlighted a list of important factors that influence Web page ranking by major SEs (SE0moz, 2009a, 2009b, 2009c, 2009d, 2009e). These factors are as follows:

- Create unique, accurate page titles,

- Make use of the "Description" meta tag,

- Improve the structure of URLs,

- Make site easier to navigate,

- Offer quality content and services,

- Write better anchor text,

- Use heading tags appropriately,

- Optimize use of images,

- Make effective use of robots.txt,

- Be aware of rel= "nofollow" for links,

- Promote Website in the right ways,

- Make use of free Webmaster tools,

- Take advantage of Web analytics services,

- Use Google Website optimizer.

The above-listed factors might differ from time to time, and this is because SE companies keep changing the way their ranking algorithms work. For this reason, every two years, SEOMoz conducts a global survey of SE optimizers, asking them questions to get an insight into how they are 
working. The 2009 results show that the top five positive ranking factors are "Anchor Text from External Links", "Keyword Use in the Title Tag", "Raw Link Popularity", "Diversity of Linking Domains" and "Keyword Use in Root Domain", whereas the top negative factors are "Link Acquisition from Link Brokers" and "Linking Out to Spam" (Searchcowboys.com, 2009). The difference between the two lists above highlights the fact that even SEO companies cannot come up with a unified list of such factors. However, by looking at the two lists above and the third list enumerated below, one can see some similarities.

Google's ranking algorithm takes into account more than 200 attributes when determining the position of Websites in SERPs (Green Marketing 2.0, 2009; Evans, 2007). According to Google (2008), the top ten factors that contribute to the elevation of a Web page in SERPs are as follows:

1. Keyword use in Title Tag,

2. Anchor Text of Inbound Links,

3. Keyword Use in Document Text,

4. Accessibility of Document,

5. Links to Document from Site-Internal Pages,

6. Primary Subject Matter of Site,

7. External Links to Linking Pages,

8. Link Popularity of Site in Topical Community,

9. Global Link Popularity of Site,

10. Keyword Spamming.

\section{Other Complex Factors Influencing Website Ranking}

Traditionally, it has been considered that the primary drivers for Website success, and thereby high ranking, are: Usability, Accessibility, SEO and Social Media/Networking (see Figure 1 below). Many researchers have written about this relationship (Baker, 2007; Compass Designs, 2011; SEWatch, 2007; Berg, 2007a; Berg, 2007b; Davies, 2009), and have showed that there were some overlaps between the four complex factors in determining site success in attracting potential customers by being visible to SEs.

Usability has always been accepted as a critical contributor in the success of a Website in that if users cannot easily find what they are looking for, they will leave and go elsewhere. Jakob Nielsen puts this succinctly saying: "Usability rules the Web. Simply stated, if the customer can't find a product, then he or she will not buy it." (Neilsen, 1999), and "The Web is the ultimate customer-empowering environment. He or she who clicks the mouse gets to decide everything. It is so easy to go elsewhere; all the competitors in the world are but a mouse click away" (Nielsen, 1999).

Accessibility is especially important for viewers with vision impairments, whether blind, color blind, elderly or simply viewing the site on a PDA/mobile phone with limited screen size. This means that Websites must be well laid-out both in terms of organization (semantic layout) as well as graphics, space, text and font effects. Performance is also an issue. Viewers on slow computers or with low speed connections (dial-up is still used) need simple sites that use minimal graphics or Flash (otherwise users might decide to go away rather than wait for a beautiful, feature- rich, Web page to load). Furthermore, some users, for various reasons, will browse a site with Java and/or JavaScript turned off. All of these groups need an accessible Website (Compass Designs, 2011).

Even with a highly accessible Website, however, people need to be able to find it, and this means using a SE. A Website with no traffic stands with little chance of achieving its goals. Probably, the most effective way to get traffic is through SEO. Unfortunately, "build it and they will come" is not true on the Web.

Furthermore, with increasing development in Web 2.0, new concepts and ideas are being explored, among which are Social Media and Social Networking. Both can help to increase Website traffic. Social Media can be used to direct traffic to organizational Websites; for example, Web blogs, now 
found at many online sites, may be syndicated through Web feeds. It is also possible to combine SEO and Social Media to promote Websites, obtaining benefits from both. In this case, SEO helps to market organizational Websites, including their Social Media content which, in turn, assists by generating referral traffic from, and credibility with, various SEs (Online Social Networking, 2011; Jackson, 2008; Jackson, 2009). For example, Del.icio.us is a social bookmarking site that enables anyone to bookmark a page. Because of its popularity and the fact that a bookmark can be interpreted as an implicit recommendation of a page, the more people who bookmark a specific page, the more chance there is to increase the page's ranking (Evans, 2007).

In summary, for a Website to achieve its goals (and a successful Website must have goals to give it direction and focus), it is not sufficient to have good ideas and good intentions. The Website needs to meet the following multi-faceted characteristics: a) Usability; b) Accessibility; c) Search Engine Optimized; d) Social Media/Networking Enabled. In many cases, there is overlap between these factors. This overlap can be represented graphically, as in Figure 1 (Compass Designs, 2011):

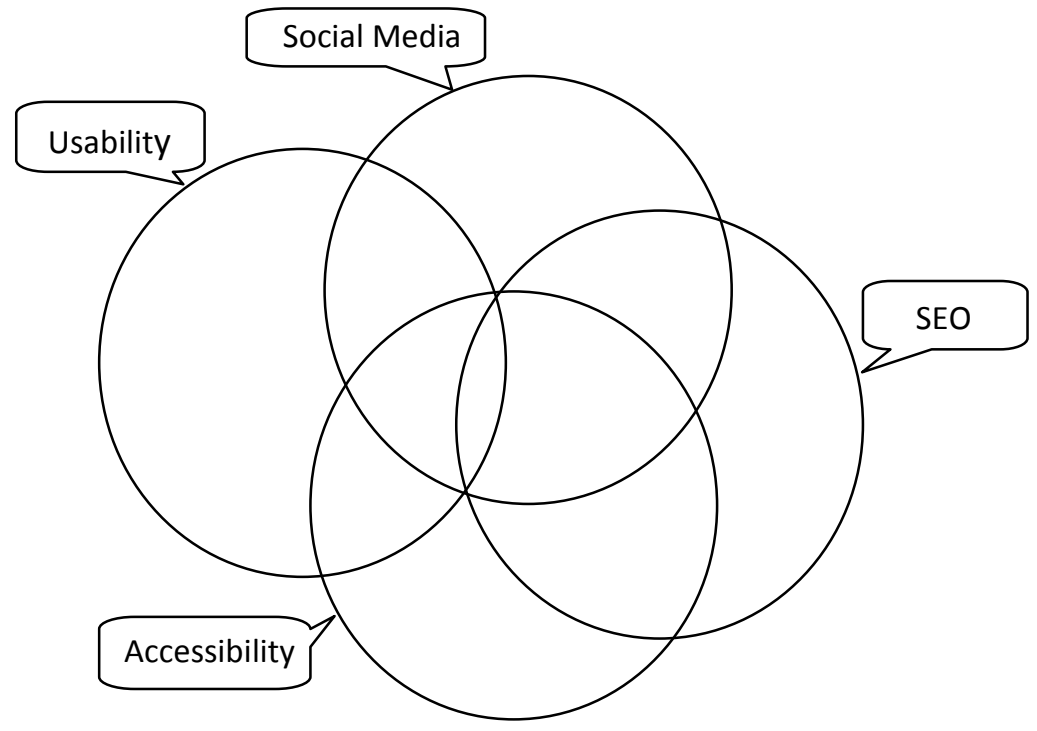

Figure 1: Overlapping between the Four Major Characteristics of Good Website

As a final word on this topic, it may be appropriate to mention the issue of standards compliance. Web browsers from different vendors have their own quirks and peculiarities. Because of this, a Website may perform well with one browser (say Internet Explorer), but badly with another (say Google Chrome). To ensure standards compliance and maximize portability between operating systems and browsers, a site can be coded to be standards compliant, and this can be validated using the W3C Markup Validation Service (W3C, 2011).

\section{Research Methodology}

Until recently, many webmasters/designers including the authors thought that adding Meta keywords tags to Web pages was the only way to increase traffic to Web pages, elevating their rankings. For this experiment, all possible keywords were included in the Meta tags but it was found that this does not improve the Website ranking. Hence, it was decided to look for other ways to improve the ranking of Web pages. For this purpose, researchers, some of whom are also Webmasters at the Sultan Qaboos University (SQU), conducted a review of SEO, exploring the different guidelines, tools, techniques and strategies that help to improve the ranking of Websites. During this exploratory study, it was discovered that there are many tools and guidelines that are useful in improving page ranking. The most prominent ones are from Google, some of which are: 
a. Google Webmaster Guidelines (Google, 2009d): these provide detailed reports about a page's visibility on Google.

b. Google Analytics (Google, 2009e): this basically shows what is happening on the Web site, and displays data similar to Google Webmaster Tools.

c. Google Website Optimizer (Google, 2009b): this provides the tools needed to improve the Website.

d. Google Trends (Google, 2009f): webmasters use these to compare different search terms.

e. Meta tag analyzer (SEOCentro, 2009): this is used to analyze the different Meta tags within a Website; researchers conducted a comparison between the SQU Website and highly ranked Websites in terms of Meta tag keywords, description, title etc.

f. SEOmoz tools (SEOmoz, 2009f): the SEOmoz toolset includes over twenty SEO tools designed to help with every aspect of SEO, including on-page targeting, site crawlability, competitive analysis, ranking, checking and keyword difficulty.

Some of these tools required a user subscription in order to be used. To explore further the effectiveness of these tools and guidelines, an Action Research project was undertaken. Action research is "learning by doing" - a group of people identify a problem, do something to resolve it, see how successful their efforts are, and if not satisfied, try again (O'Brien, 1998). The researchers at SQU modified the SQU Website as recommended by the guidelines to improve the design using the available tools as stated above. The SEs were given sometime to gather the new update and index it, before checking to see if the Website's ranking had changed. This process was repeated several times; that is, some changes were implemented as recommended by one of the tools or guidelines stated above, then their impact on the page ranking was checked using Google SE. In this experiment, all the above tools/guidelines were used, one at a time, to measure the promised impact on ranking.
The following section provides more indepth information on the actions taken in this on-going project.

\section{Findings and Results}

To enable the impact of optimization methods to be determined, phased changes suggested by the research, and based on feedback from SEO planning tools, were made to the SQU Website. These changes involved modifying the text on pages and in tags. Some of the changes made are described below:

- Page titles were changed to fit recommended lengths, and made more meaningful and descriptive. Appropriate keywords and search terms were added.

- Duplicated page titles were removed or changed to be more representative of page content.

- Page descriptions were changed to fit recommended lengths, and made more representative of page content. Appropriate keywords and search terms were added.

- Duplicate page descriptions were removed or changed to properly reflect page content.

- Pages containing errors were corrected or, if not wanted, removed.

- Links to non-existent pages were fixed; either a corresponding page was created or the link was removed.

- Image-only section headings were replaced by text, and then marked as headings.

- Where omitted, an "alt tag" was added to the images in pages (when present).

- Where keywords were included in the keyword Meta tag alone, they were added in other places too, including page title, page description, page body, heading tags etc.

As SE rankings do not change immediately, but depend upon the indexing of Web crawlers, time was allowed for SEs updates. 
Generally, it was necessary to wait several days to be assured of updates being properly recognized and included in results.

Following the update, the site's rankings and traffic were measured using the tools mentioned above. Improvements in hits driven to the Website through SE references (as opposed to direct or indirect referral) were taken to be a measure of more effective SE access and ranking.

To further examine and illustrate one of the important points mentioned in the guidelines, which is the importance of the "title" tag, the researchers conducted the following small experiment. First we searched for the term "SQU CIS home page", and the SE returned some pages related to our site but the intended page did not appear on top of the list. The reason was that the page title did not include "CIS", so this was added. After waiting for some time to allow the SE to update its tables, the search was repeated - the intended page was at the top of SERPs.

The researchers acknowledge that attempting to measure the impact of a dynamically changing resources, such as a $\mathrm{SE}$, especially in the context of the Internet, poses certain fundamental difficulties. It is rather like trying to hit the proverbial moving target. Nevertheless, it is felt that the changes made were effective. That is; using these guidelines and tools did indeed improve the ranking of the SQU Website but, as this research is ongoing, the authors are still experimenting with these tools and guidelines. Some interim data can be shown here, based on work to improve the SQU Website's visibility.

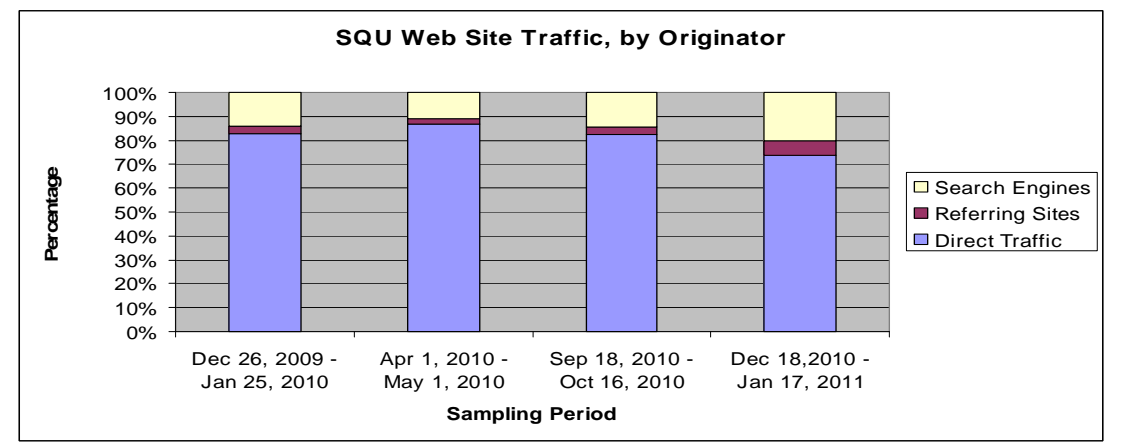

Figure 2: SQU Website Traffic, by Originator (from Google Analytics)

As can be seen, over the course of one year, the SQU Website has shown a significant improvement in its visibility for SEs, which means that the tools and guidelines used in this experiment were effective and yielded great benefits in increasing the Web pages' ranking.

\section{Conclusions and Recommendations}

Poorly-ranked sites are either deficient in quality, accessibility, usability, content, and/or inappropriateness for target audience. These issues, in addition to the aesthetics and functionality of a Website, need to be considered in order to increase the visibility, searchability and ranking of a Web page.

Different SEs do not work the same way, resulting in something that is ranked high in one not being so well placed in another. SEs use a wide variety of factors to determine site rankings, and many SEO companies conduct different surveys at different intervals of times in an attempt to predict the factors that the SE companies use in their searching algorithms. Hence, to maximize page ranking, especially for a number of different SEs, one needs to carefully balance a complex set of factors. It is very important to consider all possible ranking factors and attempt to optimize the Website to maximize SEs visibility. Meta tags are not the only factors that drive SE hits, and thereby Website ranking. Reliance on meta-tags solely is not the way to achieve high rankings (Google, 2009a; 2008; 2009c; huomah.com, 2009; Whalen, 2001; Lewis, 2009; Web.com, 2011). 
There are many guidelines for improving visibility and elevating the ranking of a Website. Designers/developers need to follow published guidelines and use available tools to enhance Websites visibility. The guidelines and tools that are provided by SEs such as Google are very useful, as seen in this experiment.

Designers need to keep up-to-date with the latest Web design and try to use them in their sites. Today Web 2.0 and Social Media/Networking are important drivers which can be used to increase Website traffics. Moreover, SE companies know the ways in which unscrupulous designers try to deceive SEs in order to achieve higher rankings than deserved (Mihm, 2011). Therefore, they continuously refine their systems and algorithms to combat such attempts to manipulate and trick them.

\section{Acknowledgement}

The authors would like to express their appreciation and gratitude to Stephen Millmore, from Sultan Qaboos University, Center for Information Systems, for his valuable comments and suggestions during the writing of this paper.

\section{References}

Baker, T. J. (2007). "Usability, Accessibility, Web Standards and SEO," accessed on 7/1/2011, available at: http://www.joomlashack.com/tutorials/ 159-usability-accessibility-web-standardsand-seo

Battelle, J. (2006). “The Search: How Google and Its Rivals Rewrote the Rules of Business and Transformed Our Culture," Portfolio Trade, ISBN-10: 1591841410

Berg, K. K., (2007a)."Usability and SEO," accessed on 16/1/2011, available at: http://blog.searchenginewatch.com/071 022-10513

Berg, K. K. (2007b). "Information Architecture: The Backbone of SEO \& Usability," accessed on 6/6/2011, available at:

[http://searchengineland.com/informationarchitecture-the-backbone-of-seo-usability30658]

Bifet, A., Castillo, C., Chirita, P.-A. \& Weber, I. (2005). "An Analysis of Factors Used in
Search Engine Ranking," Proceedings of the Workshop on Adversarial IR on the web, Chiba, 10-14 May.

Compass Designs (2011). "Usability, Accessibility, Web Standards, SEO and You," accessed on 6/6/2011, available at: http://www.compassdesigns.net/joomla -tutorials/usability-accessibility-webstandards-seo

Davies, G. (2009). "SEO, Usability \& 15 Stupid Things You Can Do to Your Web site - SMX West," accessed on 6/6/2011, available at: http://www.marketingpilgrim.com/200 9/03/seo-usability-15-stupid-things-youcan-do-to-your-Website-smx-west.html

Deitel, P. J. \& Deitel, H. M. (2008). "Internet and World Wide Web How to Program," Pearson Prentice Hall, 4th edition

Evans, M. P. (2007). "Analysing Google Rankings through Search Engine Optimization Data," Internet Research, Vol. 17 No. 1, 2007, pp. 21-37

Fleischner, M. H. (2011). SEO Made Simple: Strategies for Dominating the World's Largest Search Engine, 2nd edition, ISBN10: 1460908511

Fortunato, S., Boguna, M., Flammini, A. \& Menczer, F. (2006). "How to Make the Top Ten: Approximating PageRank from Indegree," the 14th World Wide Web Conference, Edinburgh, May 22-26, available at:

http://arxiv.org/PS_cache/cs/pdf/0511/05 11016v1.pdf

Google, (2008). "Search Engine Optimization Starter Guide," accessed on 6/6/2011, available at http://static.googleusercontent.com/exte rnal_content/untrusted_dlcp/www.google.c om/

en//webmasters/docs/search-engineoptimization-starter-guide.pdf

Google (2009a). “Google Basics,” accessed on 6/6/2011, available at: http://www.google.com/support/webm asters/bin/answer.py?hl=en\&answer=7089 $7 \& \mathrm{rd}=1$

Google (2009b). "Google Web site Optimizer," accessed on 6/6/2011, available at: 
[https://www.google.com/accounts/Servic eLogin?service=websiteoptimizer $\&$ continue $=$

http://www.google.com/analytics/siteopt/ \%3Fhl\%3Den\&hl=en]

Google (2009c). 'How to Read Search Results (relevant to page title, description and URL),' accessed on 6/6/2011, available at: http://www.google.com/support/Webse arch/bin/answer.py?hl=en\&answer $=13447$ 9\#search_results

Google (2009d). "Webmaster Guidelines," accessed on 6/6/2011, available at: [http://www.google.com/support/webmas ters/bin/answer.py?answer=35769]

Google (2009e). "Google Analytics," accessed on 6/6/2011, available at: http://www.google.com/analytics

Google (2009f). "Google Trends," accessed on 6/6/2011, available at: http://www.google.com/trends

Green Marketing 2.0 (2009). "Google's Top Search Engine Ranking Factors," accessed on 6/6/2011, available at [http://lornali.com/online-

marketing/seo/googles-top-search-engineranking-factors]

Huomah.com (2009). "Guide to Search Engine Ranking Factors," accessed on 6/6/2011, available at: [http://www.huomah.com/SearchEngines/Search-Engine-

Optimization/Guide-to-Search-Engine-

Ranking-Factors.html]

Interleado (2009). "SEO Software - Get More Traffic to your Web site," accessed on 6/6/2011, available at: [http://www.interleado.com/?gclid=CN6p7 e3UxZsCFRWbnAodYQHJBg]

Jackson, M. (2008). "Social Media and SEO -Friends with Benefits?," accessed on 7/1/2011, available at: [http://searchenginewatch.com/3632196]

Jackson, M. (2009). "Redesigning Your Web Site? Don't Neglect SEO," accessed on 6/6/2011, available at [http://searchenginewatch.com/3634964]
Kent, P. (2008). "Search Engine Optimization for Dummies Publisher: For Dummies," 3rd edition (June 10, 2008). ISBN-10: 0470262702, ISBN-13: 9780470262702

Khaki Sedigh, A. \& Roudaki, M. (2003). "Identification of the Dynamics of the Google Ranking Algorithm," the 13th IFAC Symposium on System Identification, accessed on 6/6/2011, available at: http://www.iranseo.com/articles/googl e_ranking_algorithm.pdf

Langlois, M. (2010). "Content is King, Quality is Queen", accessed on 6/6/2011, available http://gamertherapist.com/blog/2010/11/ $13 /$ content-is-king-quality-is-queen

Ledford, J. L. (2009). SEO: Search Engine Optimization Bible, 2nd edition, Wiley Publishing, 2009, ISBN: 0470452641 9780470452646

Lewis, S. (2009). "Links Top List of Search Engine Ranking Factors," accessed on 6/6/2011, available at [http://searchenginewatch.com/3634919]

Mihm, D. (2011). "Local Search Ranking Factors," accessed on 6/6/2011, available at http://www.davidmihm.com/localsearch-ranking-factors.shtml

Moran, M. \& Hunt, B. (2006). "Search Engine Marketing, Inc.: Driving Search Traffic to your Company's Web Site," IBM Press, Armonk, NY.

Nielsen, J. (1999). 'Designing Web Usability: The Practice of Simplicity,' New Riders Publishing.

O'Brien, R. (1998). "An Overview of the Methodological Approach of Action Research," accessed on 16/2/2011, available at :http://www.web.net/ robrien/papers/arf inal.html

Online Social Networking, (2011). "Social Media vs. Search Engine Optimization," accessed on 16/1/2011, available at: [http://online-socialnetworking.com/social-media-vs-searchengine-optimization 
Pringle, G., Allison L., and Dowe D.L., (1998). "What is a Tall Poppy among Web Pages?", Proceedings of the 7th International World Wide Web Conference, Brisbane, April, pp. 369-377, available at: http://www.csse.monash.edu.au/ lloyd /tilde/InterNet/Search/1998_WWW7.html

Searchcowboys.com (2009). "The Search Engine Ranking Factors 2009: Let's Build Links," accessed on 6/6/2011, available at: http://www.searchcowboys.com/research/ 926

SEOaddress.com (2011). 'SEO in 4 Hours, Google, Yahoo \& MSN Bing! SEO Company,' accessed on 16/1/2011, available at: http://www.seoaddress.com/index.html

SeoCentro (2009). "Meta Tag Analyzer," accessed on 24/9/2009, available at: [http://www.seocentro.com/tools/searchengines/metatag-analyzer.html]

SEOmoz (2009a). "SEOmoz: PRO Training Seminar 2009," accessed on 6/6/2011, available at:

[http://www.seomoz.org/seminar/series]

SEOmoz (2009b). "Search Engine Ranking Factors V2," accessed on 6/6/2011, available at:

[http://www.seomoz.org/article/searchranking-factors]

SEOmoz (2009c). "Search Engine Ranking Factors 2005," accessed on 24/9/2009, available

at: http://www.seomoz.org/article/searchranking-factors-2005

SEOmoz (2009d). "The Beginner's Checklist for Learning SEO," accessed on 6/6/2011, available at:

[http://www.seomoz.org/blog/thebeginners-checklist-for-learning-seo]

SEOmoz (2009e). "The Web Developer's SEO Cheat Sheet," accessed on 24/9/2009, available at:

[http://www.seomoz.org/blog/the-Webdevelopers-seo-cheat-sheet]

SEOmoz (2009f). "SEO Tools," accessed on 6/6/2011, available at:

[http://www.seomoz.org/tools]
SEWatch (2007). "Usability and SEO. Which comes First?," accessed on 16/1/2011, available

at:

[http://blog.searchenginewatch.com/07090 6-083601]

Sullivan, D. (2002). "Death of a Meta Tag," accessed on 6/6/2011, available at: [http://searchenginewatch.com/2165061]

Sullivan, D. (2007). "Meta Keywords Tag 101: How to "Legally" Hide Words on Your Pages for Search Engines," accessed on 6/6/2011, available at: [http://searchengineland.com/metakeywords-tag-101-how-to-legally-hidewords-on-your-pages-for-search-engines12099]

The Lost Agency, (2009). 'Web site Visitor Analysis,' accessed on 6/6/2011, available at:

[http://www.thelostagency.com/index.php /analytics-services/google-analytics/Webvisitor-analysis.html]

Whalen, J. (2001). “Can Meta Tags Such as the Keyword Tag Bring High Rankings to my Site?," accessed on 6/6/2011, available at: [http://www.highrankings.com/metakeyw ord]

Wilding, R. (2009). "Top 5 White Hat and Black Hat Search Optimization Techniques," accessed on 6/6/2011, available at: [http://www.pushon.co.uk/articles/top-5white-hat-and-black-hat-searchoptimisation-techniques/]

W3C (2011). "The W3C Markup Validation Service," accessed on 6/6/2011, available at:

[http://validator.w3.org/

Web.com (2011). "Search Engine Optimization - Natural Search Optimization \& SE0," accessed on 6/6/2011, available at: [http://www.submitawebsite.com/] 